\title{
ADOPCIÓN DE DOS SISTEMAS PRODUCTIVOS ADAPTATIVOS: GRANOS BÁSICOS CON ÁRBOLES DISPERSOS Y GRANOS BÁSICOS EN CALLEJONES MEJORADOS EN LA ZONA SECA DE NICARAGUA, 2006-2008
}

\section{ADOPTION OF TWO ADAPTIVE PRODUCTION SYSTEMS: BASIC GRAINS THRU SCATTERED TREES AND BASIC GRAIN AT IMPROVED ALLEYS CROPPING SYSTEMS IN NICARAGUA DRY ZONE, 2006-2008}

\author{
Toruño Pedro José1, Mendoza Ríos Carlos Ernesto² \\ ${ }^{1}$ Master of Science. Profesor e investigador de la Universidad Nacional Autónoma de Nicaragua, León (UNAN-León), km. 1 1 1/2 Carretera \\ León-La Ceiba.pjoseto@unanleon.edu.ni \\ 22Ing. Agroecólogo. carlosmr19875@gmail.com
}

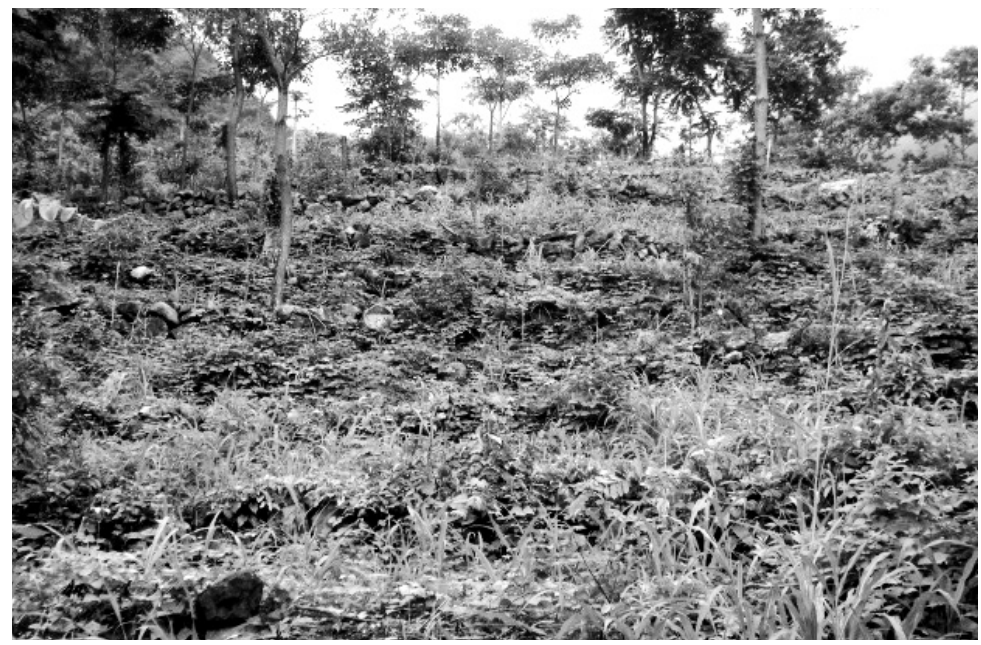

\section{RESUMEN}

En la actualidad no existe ningún registro de estudios de aceptación y/o adopción de sistemas agroforestales o bien sobre las obras físicas, prácticas y tecnologías de conservación de suelo y agua de parte de agricultores que tienen establecidos estos sistemas de producción de alguno de los siete municipios de la zona seca del occidente de Nicaragua, de ahí la necesidad de realizar estudios de aceptación y adopción en la zona. Los objetivos del estudio fueron describir las obras agronómicas y físicas de conservación de suelo más utilizadas por los agricultores en los sistemas productivos adaptativos de Granos Básicos con Árboles Dispersos (GBAD), y Granos Básicos en Callejones Mejorados (GBCM), y evaluar el grado de aceptación y adopción de los dos sistemas productivos adaptativos promovidos por el Proyecto Manejo Sostenible de la Tierra (MST); y ejecutado por las familias productoras de siete municipios de la zona seca de León, Chinandega y un municipio de Managua en el período 2006-2008. Este estudio es descriptivo y de corte transversal con valoraciones cualitativas y cuantitativas en relación al uso actual y potencial de los sistemas productivos. La metodología de investigación utilizada fue la del análisis de la toma de decisiones por medio del método rápido de sistemas diversos. Dicha metodología está diseñada para tomar decisiones utilizando indicadores sencillos para priorizar los problemas a nivel de finca con

\begin{abstract}
Today there are no records of studies of acceptance and / or adoption of agroforestry systems, as well as, practices and technologies of soil and water conservation by farmers who have established such systems in the dry region of western Nicaragua, hence the need for acceptance and adoption studies in the area. The objectives of the study were to describe the agronomic and physical soil conservation practices most used by farmers in adaptive production systems of basic grains with scattered trees (GBAD), and Enhanced Basic Grains in Alleys (GBCM), and assess the acceptance and adoption of the two adaptive production systems promoted by the project Sustainable Land Management, executed by farming families from seven municipalities in the dry zone of León, Chinandega and Managua municipality in 2006-2008. This study is descriptive and cross-sectional with quantitative and qualitative assessments in relation to current and potential use of the production systems. The research methodology used was analysis of decision making through rapid method for various systems. This methodology is designed to make decisions using simple indicators to prioritize the problems at the farm level based on three criteria: priority, management capacity and disposition of the environment. The methodology of the Hillside Sustainable Agriculture program in Central America (PASOLAC by its Spanish acronyms) was used to assess the degree of acceptance
\end{abstract}


base a tres criterios: prioridad, capacidad de manejo y disposición del medio. Para evaluar el grado de aceptación y adopción se utilizó la metodología del Programa para la Agricultura Sostenible en Laderas de Centro América PASOLAC. Los agricultores que poseen grandes extensiones de tierra fueron los que menos adoptaron y aceptaron las tecnologías difundidas, mientras que agricultores con fincas de menor extensión territorial mostraron mayor grado de aceptación y adopción de los Sistemas Agroforestales (SAF). Los organismos capacitadores que han tenido mayor presencia en las zonas de estudio son el Instituto Nicaragüense de Tecnología Agropecuaria (INTA), junto al proyecto MST, estos han ido de la mano para la divulgación de las nuevas tecnologías. Menor presencia tuvo el organismo Unidad Ambiental (UAM). La adopción de ambos sistemas agroforestales fue de $100 \%$ en casi todos los municipios. Un consolidado de la adopción de todos los municipios muestra que $73 \%$ de los mismos han adoptado ambos sistemas (GBAD y GBCM). Un alto porcentaje de los agricultores encuestados mantienen las tecnologías promovidas, expandiendo así ambos sistemas agroforestales en sus parcelas productivas, logrando un manejo sostenible de la tierra.

Palabras clave: sistemas productivos en laderas, adopción de tecnologías, sistemas agroforestales

Abreviaturas: GBAD, Granos Básicos con Árboles Dispersos, GBCM, Granos Básicos en Callejones Mejorados, SAF, Sistemas Agroforestales, MST, Manejo Sostenible de la Tierra.

M ás del $80 \%$ de la población de Nicaragua (116 de 156 municipios) se concentra en la zona seca. Esta región se caracteriza por sus suelos volcánicos fértiles, con un alto potencial para la agricultura, la ganadería y la forestería. La producción agropecuaria de la zona seca mantiene a la nación, ofreciendo más del $60 \%$ del empleo nacional y el $55.8 \%$ de las exportaciones totales (MARENA-PNUD, 2003).

La asociación del árbol con cultivos agrícolas proporcionan beneficios, ya que estos interactúan entre si obteniéndose forraje, frutos, maderas, leña, protección y aumento en la fertilidad de los suelos, trayendo como resultado una productividad de manera continuada y sostenible de todos los recursos involucrados en el sistema establecido (Montagnini, 1992). El término Sistemas Agroforestales, se usa para todos los sistemas y prácticas de uso de la tierra, donde árboles o arbustos perennes leñosos son deliberadamente sembrados en las misma unidad de manejo de la tierra con cultivos agrícolas y/o animales, tanto en mezcla espacial o en secuencia temporal; presentando interacciones ecológicas y económicas significativas entre los componentes leñosos y no leñosos (Ospina, 2007).

Los sistemas agroforestales se clasifican en varios tipos, entre ellos, el sistema de callejones mejorados y el sistema de árboles dispersos; los beneficios en el campo son muchos. Estos sistemas requieren de ciertas condiciones para su establecimiento, para así llegar a obtener el máximo de su potencial de beneficios una vez establecidos en el campo. and adoption. Farmers who own large tracts of land were those that least adopted and accepted the new technologies, while farmers with smaller land area farms showed greater acceptance and adoption of the system. Trainers agencies which had significant presence in the study areas were the Institute of Agricultural Technology (INTA), as well as the MST project, both have worked together for the dissemination of the new technologies. The Environmental Unit (UAM) group showed lower presence in the area of study. Adoption of agroforestry systems was $100 \%$ in all municipalities, a consolidated figure for all municipalities shows that $73 \%$ of the surveyed population has managed to adopt production systems of GBCM and GBAD, thereby improving production and conserving soil moisture. The agronomic and physical soil conservation practices most used by farmers are minimum tillage, cover crops, contour lines and no burning.

Keywords: hillside production systems, technology adoption, agroforestry

Abbreviations: GBAD, basic grains with scattered trees, GBCM, Enhanced Basic Grains in alleys, SAF, agroforestry, MST, ustainable land management.

La adaptabilidad de los mismos dependerá del tipo de terreno en donde están establecidos, así como también el área de ejecución, el manejo, condiciones climáticas, entre otros factores. Muchos campesinos de la zona norte de León, Chinandega y Managua dicen adaptarse bien a este tipo de prácticas agroforestales en sus fincas, aunque otros opinan lo contrario, es sabido que todo procedimiento tiene sus preeminencias y desventajas; así como también la aceptación de los sistemas varía según las condiciones dadas en las distintas zonas de ejecución.

Los sistemas de producción agroforestales han sido adoptados por pequeños y medianos agricultores al haber sido introducidos en sus fincas. Esto condujo a estos agricultores hacia la agroforesteria productiva de plantaciones forestales en combinación con los cultivos agrícolas. Este mérito es más relevante aún si se tiene en cuenta que para esta adopción se utilizó el crédito rural como herramienta de apoyo financiero al agricultor y se tiene en cuenta las condiciones climáticas adversas, los cambios institucionales efectuados y las políticas de uso de la tierra adversas a la agroforesteria productiva que predominaron durante el periodo 1989-1999 (Toruño, 2001).

En la actualidad no existe ningún registro de estudios de aceptación y/o adopción de los SAF o bien sobre las obras físicas, prácticas y tecnologías de conservación de suelo y agua más utilizadas por los agricultores que tienen establecidos estos sistemas de producción de alguno de los siete municipios donde se realizó este trabajo de investigación; de ahí la necesidad de realizar estudios de aceptación y adopción en dichos municipios. 
En este trabajo se realiza una evaluación del grado de aceptación y adopción de dos sistemas productivos adaptativos (GBCM y GBAD) impulsados por el Proyecto Manejo Sostenible de la Tierra en el período 2006 - 2008 ejecutado en siete municipios de la zona seca de León, Chinandega y un municipio de Managua. Además se da a conocer sobre la situación de los agricultores que han establecido, abandonado o continúan con estas tecnologías.

Los objetivos son: describir los sistemas productivos adaptativos GBCM y GBAD con sus obras agronómicas y físicas de conservación de suelos y agua más utilizadas por los agricultores en la parcela y evaluar el grado de aceptación y adopción de ambos sistemas.

\section{MATERIALES Y MÉTODOS}

El tipo de estudio es descriptivo y de corte transversal, haciendo valoraciones cualitativas y cuantitativas en relación al uso actual y potencial de los sistemas productivos. Se evaluó el grado de aceptación y adopción de dos Sistemas Productivos Adaptativos (GBCM y GBAD) impulsados por el Proyecto Manejo Sostenible de la Tierra (MST) en el período 2006 - 2008 ejecutado por familias productoras de siete municipios de la Zona Seca de León, Chinandega y un municipio de Managua, que introdujeron el Sistema Agroforestal en su parcela productiva en el año 2006.

La metodología de investigación utilizada fue la del análisis de la toma de decisiones por medio del método rápido de sistemas diversos propuesto por Mora et al., (2004), dicha metodología está diseñada para tomar decisiones utilizando indicadores sencillos, para priorizar los problemas a nivel de finca sobre la base de tres criterios: Prioridad, Capacidad de Manejo y Disposición del Medio. Para evaluar el grado de aceptación y adopción se utilizó la metodología de PASOLAC plasmada en la Guía Técnica de Conservación de Suelo y Agua (2005).

El universo total es de 79 agricultores, localizados en los siguientes municipios; Cinco Pinos-Somotillo: 10 agricultores, San Francisco del Norte: seis agricultores, Achuapa: tres agricultores, El Sauce: 15 agricultores, Santa Rosa del Peñón: 10 agricultores, San Francisco Libre: 30 agricultores y El Jicaral cinco agricultores.

La selección de los agricultores se hizo de manera aleatoria siguiendo la muestra del $20 \%$ del total de la población, dividiéndola entre el total de agricultores con el sistema de GBAD y GBCM para así obtener la muestra de agricultores por sistema productivo.

La muestra de este estudio corresponde a 10 agricultores que tienen establecido el sistema de granos básicos con árboles dispersos (GBAD) y cinco agricultores con el sistema de granos básicos en callejones mejorados (GBCM), dando un total de 15 agricultores, lo cual representa el $20 \%$ de la muestra. Estos agricultores corresponden a los que iniciaron el establecimiento del sistema productivo (GBAD y/o GBCM) en el año 2006 para ser evaluados durante el periodo 2006-2008.
El análisis estadístico de los indicadores técnicos se realizó mediante el uso de estadística descriptiva (promedio, máximo, mínimo e intervalos de frecuencia), utilizando hojas de cálculo para el procesamiento de los datos, Microsoft Excel 2007.

\section{RESULTADOS Y DISCUSIÓN}

Características de los agricultores. Es notorio que el sexo dominante entre los encuestados es el masculino con un $93 \%$ del total de la muestra, siendo solo el $7 \%$ que corresponde a una persona del sexo femenino. La adopción de tecnologías difiere muchas veces entre hombres y mujeres, estos datos pueden explicar diferencias en acceso a créditos, mercado, fuerza de mano de obra, responsabilidades y derechos en la finca, en este caso, dada la mínima participación de la mujer (correspondiente en este estudio) no hay una incidencia significativa y comparativa para poder afirmar que existió diferencia en la adopción y/o aceptación del SAF.

Nivel de educación de los agricultores. Al analizar la información de la tabla 1 correspondiente al nivel de escolaridad de los agricultores sujetos de estudio, se encontró que el $26.7 \%$ de ellos son analfabetos, no obstante, siendo un porcentaje un poco elevado, los agricultores lograron apropiarse de las tecnologías difundidas por el proyecto MST, logrando solo un $63.7 \%$ del porcentaje total de los agricultores analfabetas que no lograron adoptar el SAF en sus parcelas productivas. Seguidamente identificamos que el $53.3 \%$ de los encuestados llegaron a la educación primaria, pero sin concluirla, de este porcentaje, el 33.3\% logró la adopción de las tecnologías, mientras que el $20 \%$ no adoptaron el SAF; sin embargo, el $20 \%$ restante de los agricultores tienen un nivel educativo de secundaria incompleta, logrando un $100 \%$ de adopción entre este nivel educativo. El nivel de educación afecta el entendimiento del material didáctico y las capacitaciones impartidas a los agricultores.

Tabla 1. Nivel de educación de los agricultores

\begin{tabular}{lccr}
\hline $\begin{array}{c}\text { Nivel } \\
\text { de educación }\end{array}$ & Si adoptan & No adoptan & Total \\
\hline Analfabetismo & 20.0 & 63.7 & 26.7 \\
Primaria incompleta & 33.3 & 20.0 & 53.3 \\
Primaria completa & 0.0 & 0.0 & 0.0 \\
Secundaria completa & 20.0 & 0.0 & 20.0 \\
\hline Total & 73.3 & 26.7 & 100.0 \\
\hline
\end{tabular}

Edad de los agricultores. Es importante destacar que el $60 \%$ de los agricultores oscilan entre las edades de 30 a 60 años, siendo esta la edad promedio de los agricultores en esta región del país, lo que es ventajoso puesto que son personas adultas con alto grado de experiencia, fuerza de mano de obra y responsabilidad, debido a que en su mayoría son jefes 
de familia y están conscientes de las acciones realizadas en su finca y de la importancia de hacer mejoras en ellas, no obstante, la edad refleja un alto nivel de desadopción, con un $20 \%$, pero se logró un $40 \%$ de adopción de las tecnologías difundidas. La razón por la cual se dio el mayor índice de aceptación y adopción entre los campesinos con este rango de edad, es que la mayoría son originarios de los municipios donde mayor porcentaje de desadopción y desadaptación como son los municipios de San Francisco Libre y El Jicaral, mientras que solo un $26.7 \%$ de los 15 agricultores oscilan entre 60 a 90 años, considerados como los agricultores con un alto nivel de conocimiento, teniendo un índice mínimo de desadopción del $6.7 \%$ y un $20 \%$ de adopción entre este intervalo; no obstante, solo el $13.3 \%$ corresponde a agricultores que oscilan entre las edades de 19-30 años, siendo este rango de edad el que obtuvo un 100\% de adopción de los SAF en sus parcelas productivas a pesar de su edad temprana y poco conocimiento empírico en comparación con los agricultores de edades entre 60-90 años (tabla 2).

Tabla 2. Porcentaje de adopción en correspondencia con la edad de los agricultores

\begin{tabular}{lccc}
\hline $\begin{array}{c}\text { Rango } \\
\text { de edades }\end{array}$ & Si adoptaron & No $^{\circ}$ adoptaron & Total \\
\hline $19-30$ & 13.3 & 0.0 & 13.3 \\
$30-60$ & 40.0 & 20.0 & 60.0 \\
$60-90$ & 20.0 & 6.7 & 26.7 \\
\hline Total & 73.3 & 26.7 & 100.0 \\
\hline
\end{tabular}

Características de la finca

Tamaño de la finca. Más de la mitad de la población encuestada (ocho agricultores) tienen fincas pequeñas entre 1-5 manzanas (0.7 y $3.51 \mathrm{ha}$ ) y por ende, el área dedicada a la agricultura es pequeña, sin embargo, cuatro agricultores poseen más de 10 manzanas de tierra (7.03 ha), donde el área dedicada a la producción agrícola debe ser mayor, así como también los ingresos brutos, costos totales, etc., y solamente tres agricultores poseen fincas con más de 20 manzanas distribuidas en grandes lotes dedicados a la actividad agrícola, pecuaria, forestal, más el área de la finca. La hipótesis que se plantea es que a mayor área de extensión del SAF, mayor será el índice de aceptación, siendo todo lo contrario con los datos de la información obtenida, dado que los agricultores que poseen grandes extensiones de tierra fueron los que menos adoptaron y aceptaron las tecnologías difundidas, mientras que los agricultores con fincas de menor extensión territorial fueron quienes demostraron mayor grado de aceptación y adopción de los SAF.

Utilidad de la tierra. En general, la orientación productiva predominante en los siete municipios, es de carácter agrícola muy tradicional, donde cada finca cuenta con características específicas que se derivan de la diversidad existente en lo relacionado a la dotación de recursos y a las circunstancias familiares, no obstante, cada vez más la frontera agrícola avanza a un ritmo alarmante, indicando que el área forestal cada vez es menor. Un aspecto que es importante destacar son los tipos de paisajes existentes en la zona de amortiguamiento que en general evidencian un paisaje fragmentado por la fuerte actividad pastoril y agrícola, como es el caso de San Francisco del Norte, donde la mayoría de las fincas destinan más áreas a la producción pecuaria; en similares porcentajes está el municipio de El Sauce, donde el área destinada al sector pecuario es superior al agrícola, sin embargo, no todos los municipios tienen la misma distribución del área de sus fincas, tal es el caso de San Francisco Libre donde casi la mitad de las fincas son destinadas a la producción agrícola (tabla 3).

Tabla 3. Distribución (\%) de la utilidad de la tierra por municipios

\begin{tabular}{lcccc}
\hline \multicolumn{5}{c}{ Orientación productiva } \\
\hline Municipio & Agrícola & Forestal & Pecuaria & Huerto/Hogar \\
\hline $\begin{array}{l}\text { El Sauce } \\
\text { Santa Rosa }\end{array}$ & 25.3 & 24.3 & 39.2 & 11.1 \\
$\begin{array}{l}\text { del Peñón } \\
\text { San Francisco }\end{array}$ & 37.5 & 12.5 & 37.5 & 12.5 \\
$\begin{array}{l}\text { Libre } \\
\text { San Francisco }\end{array}$ & 46.4 & 26.7 & 19.0 & 7.9 \\
del Norte & 11.8 & 20.6 & 44.1 & 23.5 \\
$\begin{array}{l}\text { El Jícaral } \\
\text { Cinco pinos- }\end{array}$ & 37.5 & 12.5 & 37.5 & 12.5 \\
-Somotillo & 44.0 & 3.2 & 37.0 & 15.8 \\
\hline
\end{tabular}

Tenencia de la tierra. La tenencia de la tierra es un componente muy importante que se consideró, y es uno de los factores clave para la toma de decisiones en la aceptación y/o rechazo de una innovación tecnológica. En este caso el $93 \%$ de los agricultores entrevistados son dueños de las propiedades que habitan; este dato es muy ventajoso ya que indica que en el momento de la toma de decisión con respecto a la introducción o no de una tecnología ofrecida al productor, éste será consciente de que los resultados de la decisión que tome quedará asentada en su propiedad, apropiándose de ello y sintiéndose orgulloso de sus logros y de la decisión tomada. El 7\% (un productor) heredó la finca, pero no posee título de propiedad, concluyéndose que el 100\% de los agricultores de los distintos municipios poseen finca propia.

\section{Características del sistema de transferencia Personas involucradas en la realización y mantenimiento de las tecnologías en transferencia. La implementación de estos sistemas ha contribuido a mejorar las relaciones intrafamiliares, puesto que prácticamente toda la familia participa en la realización y mantenimiento de las obras de}


conservación de suelo y agua; así como también de los SAF, estando presente en el $58 \%$ de todas las labores realizadas en la finca, mientras que solo en el $20 \%$, el jefe de familia sin ayuda de nadie realiza todas las labores, y en el otro $20 \%$, el productor paga a un jornalero para la realización de dichas obras. El alto porcentaje de la participación familiar sin la necesidad de contratación de mano de obra externa, disminuye los costos de producción de la parcela, aumentando así el beneficio neto y teniendo mayores ingresos económicos por la disminución de los costos por pago de mano de obra, además, la familia mientras implementa las tecnologías, aprende a manejarlas y darles mantenimiento.

\section{Institución facilitadora de la capacitación de las tecnologías}

en transferencia. En lo que respecta a la asistencia técnica, los agricultores aseveran que la asistencia y capacitación fue buena en cuanto al tiempo dedicado a la misma y por lo que consideran que se cumplió satisfactoriamente el objetivo de aprender el uso de la propuesta innovadora. Es notorio que los organismos capacitadores que han tenido mayor presencia en las zonas de estudio son el Instituto Nicaragüense de Tecnología Agropecuaria (INTA) junto al proyecto MST, estos han ido de la mano en la divulgación de las nuevas tecnologías, teniendo menor presencia organismos como la Unidad Ambiental (UAM) de las alcaldías municipales y otros organismos que habían tenido presencia en años anteriores zonas de estudio. Muy importante es hacer mención de la

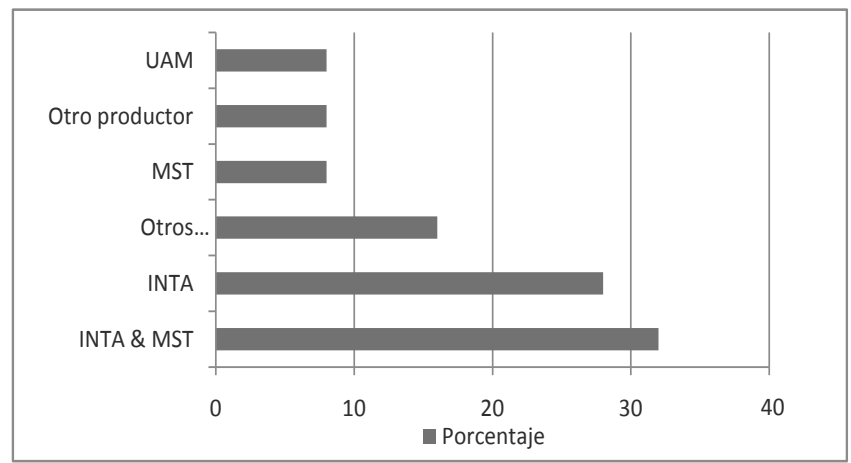

Figura 1. Instituciones facilitadoras de capacitaciones de tecnologías de transferencia.

replicabilidad en la zona, donde el $8 \%$ de los agricultores han aprendido estas tecnologías de otros agricultores, pero este índice, que es muy bajo, no se refiere a que los agricultores no divulguen estas tecnologías, sino al bajo conocimiento que tienen acerca de los SAF.

Razones que motivaron a los agricultores a la adopción de las tecnologías difundidas. La figura 2 , muestra las razones dadas por los agricultores del por qué han adoptado y aceptado los SAF y las obras de conservación de suelo y agua en sus fincas, siendo la razón principal el aumento en la producción en las parcelas donde tienen establecidas las distintas obras y tecnologías, no obstante, la mayoría de los agricultores consideran que estas obras y tecnologías no requieren de mucho dinero ya que muchos de los recursos que se utilizan para el establecimiento y mantenimiento de las tecnologías son locales, como piedras, rastrojos de cosechas etc., evitando el gasto adicional en la compra de materiales y por ende minimizando los costos de establecimiento y mantenimiento de dichas tecnologías. También, pero en baja escala, los agricultores consideran que éstas obras no requieren de mucho tiempo, ya que la mayoría consideran que las obras que requieren mayor tiempo de construcción y mantenimiento, son las de retención de suelo y agua, pero están conscientes de los beneficios que trae la implementación de éstas en sus fincas.

Los agricultores están de acuerdo en que hay que ser pacientes para ver los resultados esperados, para ello deben de esforzarse en darle continuidad y extender las distintas obras de conservación de suelo y agua; muchos agricultores que han adoptado estas tecnologías consideran que han mejorado la fertilidad del suelo, así como también la retención de la humedad. Con menor número de repeticiones, pero no menos importante, establecieron las obras de conservación y los SAF con la curiosidad de ver "qué pasa" al establecer estas tecnologías, además, otros agricultores lo hicieron por la facilidad del establecimiento de las mismas y el alto que ponen los SAF al monocultivo, saliendo de la práctica tradicional y dirigiéndose al policultivo por el beneficios que trae este sistema.

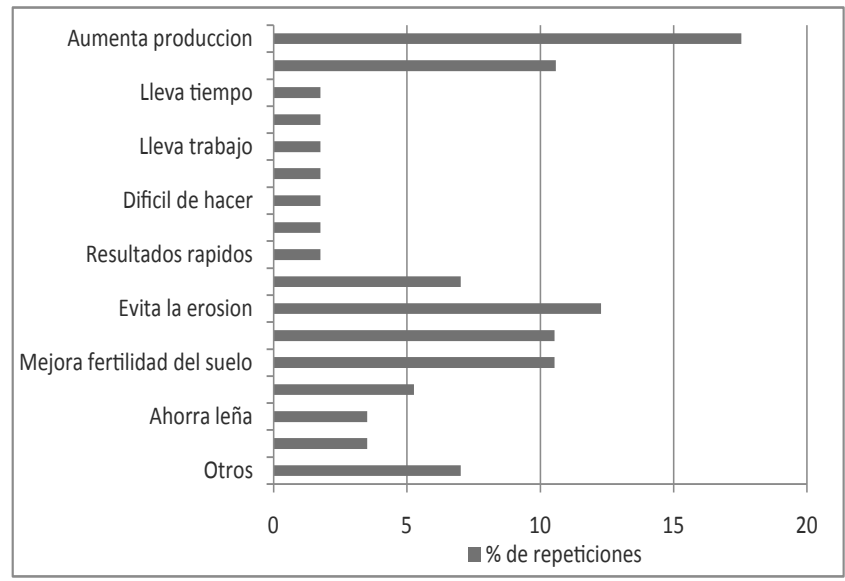

Figura 2. Razones que motivaron a los productores a la adopción y continuación de las tecnologías difundidas.

Índice de aceptabilidad de los sistemas GBCM y GBAD. Uno de los factores claves para la aceptación y adopción de una tecnología es que es el productor divulgue la tecnología que le es enseñada; con la replicación se recupera y acumula el aprendizaje que deja la experiencia. El 32\% de los agricultores entrevistados divulgan las tecnologías a otros agricultores que viven fuera de la comunidad, siendo alentador este índice ya que demuestra que las tecnologías 
que les fueron enseñadas están siendo aprendidas por personas ajenas a las zonas donde se trabaja con la adopción y aceptación de nuevas tecnologías, sin embargo, estos conocimientos no solo se distribuyen fuera de la comunidad, sino también, dentro de la misma ( $26 \%$ de los agricultores que replican las tecnologías a fincas vecinas) y dentro de la familia con un $21 \%$, valor muy similar al anterior, sin embargo, no podemos dejar excluido que el $21 \%$ de los agricultores no divulgan las tecnologías adoptadas, ya que la población vecina y los familiares ya tienen conocimiento o bien, practican estas tecnologías en sus fincas.

Claramente se ve el índice de aceptación casi igual con el porcentaje de agricultores que no han aceptado en su totalidad el sistema agroforestal que le es difundo, para que éstos lo acepten y adapten a su parcela productiva, donde el total de aceptación en todos los municipios ha sido del $51.9 \%$, mientras que el porcentaje de agricultores que no han aceptado los SAF es de $48.1 \%$, por tanto, se plantea un reto por parte de los organismos que impulsan estos sistemas que consiste en revisar la metodología de extensión que están utilizando con la finalidad de aumentar el índice de aceptación de los SAF. Es notorio que el comportamiento de la aceptación varía por cada municipio, en El Sauce donde el índice de aceptación es igual a $83.3 \%$ para el sistema GBAD y $57.1 \%$ para GBCM, hay mucho que trabajar para que el productor logre extender más el SAF difundido en éste y todos los municipios donde trabaja el proyecto MST, especialmente en el sistema de GBCM, que es quien mayor índice de no aceptación tiene en comparación con GBAD. Otros municipios han logrado excelentes índices de aceptación, tal es el caso de Santa Rosa del Peñón, Cinco Pinos-Somotillo, aunque este último se inclina más a GBAD. Igual índice sucede con San Francisco del Norte, pero con aceptación hacia GBCM, otro municipio que presenta problemas de aceptación es El Jicaral, donde apenas se logra el 33.3\% de aceptación dirigido a GBCM y $100 \%$ de no aceptación del sistema GBAD.

Con respecto al municipio de San Francisco Libre es de esperarse ese índice tan pesimista como lo es el $27.1 \%$ de aceptación en el sistema GBAD y $22.3 \%$ en el sistema de GBCM, en este municipio aparte de las irregularidades que hay con la asistencia técnica en las comunidades, existe también según los agricultores, falta de ayuda tanto material (herramientas), como educativo, ya que desconocen ciertas prácticas, y en lo cultural, porque ellos consideran como la mejor opción la práctica tradicional, ya que se sienten satisfechos con los resultados obtenidos, porque -según opinión de la mayoría de los agricultores de este municipiolos SAF requieren de mucha mano de obra, tiempo y perseverancia para ver resultados, además, consideran que existe abandono por parte de los organismos de extensión ubicados en la zona.
Nivel de adopción de los sistemas GBCM y GBAD por municipio. La adopción fue evaluada en 15 sistemas productivos distribuidos en 10 fincas de agricultores que tienen establecido el sistema de granos básicos con árboles dispersos (GBAD) y cinco agricultores con el sistema de granos básicos en callejones mejorados (GBCM), dando un total de 15 agricultores, lo cual supone el $20 \%$ de la muestra. El 80\% de las familias participantes han abandonado la quema de rastrojos. El 100\% de las familias participantes mantienen el sistema agroforestal, de las cuales el 7\% representa la aproximación de las mujeres al Proyecto. El alto porcentaje de adopción en ambos sistemas, asegura la apropiación de la tecnología y la sostenibilidad del proyecto.

El conjunto de las actividades de promoción y formación del sistema agroforestal de granos básicos en árboles dispersos y el de callejones mejorados ha contribuido a reforzar la sensibilidad ambiental de las familias.

La participación de la mujer asegura la transferencia del sistema agroforestal de generación en generación, rompiendo el patrón de extensión en la agricultura dirigida para y por los hombres.

La apropiación del sistema agroforestal GBAD y el sistema GBCM permite cambiar la cultura de quema de las familias participantes, teniendo la mujer un fuerte papel en este proceso.

El trabajo en conjunto de hombres, mujeres y niños en el establecimiento del sistema, fortalece la adopción del mismo, que además, implica el establecimiento de una reserva energética o de madera de construcción, y supone una fuente potencial de material vegetativo para usos variados, así como también la obtención de frutos tanto para alimento de la familia como para el ganado, o bien para la comercialización del mismo.

El sistema agroforestal rescata suelos altamente degradados que habían sido abandonados por su improductividad, contribuyendo además a frenar la erosión del suelo y proteger a las familias de posibles desastres socio natural a corto y largo plazo.

La adopción de los dos sistemas agroforestales (GBAD y GBCM) fue en casi todos los municipios del $100 \%$. El consolidado de la adopción de las tecnologías nos demuestra que $73 \%$ de los municipios han adoptado ambos sistemas (GBAD y GBCM) y un $27 \%$ de desadopción distribuidos en ambos sistemas. Muchos de los municipios presentan altos niveles de adopción en ambos SAF, el único municipio que tiene problemas con la adopción de los sistema GBAD y GBCM es San Francisco Libre, donde existen ciertos inconvenientes con la asistencia técnica, irregularidades con las precipitaciones (meses que llueve demasiado y otros que llueve muy poco), además tienen irregularidades con la entrega de materiales hacia los agricultores. La tabla 4 indica que en el municipio de San Francisco Libre, existe un 40\% de adopción de GBAD y $60 \%$ de desadopción, no aceptando en un $100 \%$ la tecnología de GBCM. 
Tabla 4. Nivel de adopción de los sistemas GBCM y GBAD por municipio

\begin{tabular}{lcccc} 
Municipio & $\begin{array}{c}\text { Si adoptaron } \\
\text { GBAD }\end{array}$ & $\begin{array}{c}\text { Si adoptaron } \\
\text { GBCM }\end{array}$ & $\begin{array}{c}\text { No adoptaron } \\
\text { GBAD }\end{array}$ & $\begin{array}{c}\text { No adoptaron } \\
\text { GBCM }\end{array}$ \\
\hline El Sauce & 100 & 100 & 0 & 0 \\
Santa Rosa del Peñón & 100 & 100 & 0 & 0 \\
San Francisco Libre & 40 & 0 & 60 & 100 \\
San Francisco del Norte & 100 & 0 & 0 & 0 \\
El Jícaral & 0 & 100 & 0 & 0 \\
Cinco pinos / Somotillo & 100 & 100 & 0 & 0 \\
& & & & \\
\hline
\end{tabular}

Promedio

73

27 suelo se vuelve sensiblemente más importante en la producción orgánica, porque en su manejo ya no se le considera como únicamente materia, sino por el contrario, se trata de un "organismo vivo" y requiere por tanto, todos los cuidados y consideraciones necesarias para su utilización desde un punto de vista sostenible.

Los agricultores entrevistados realizan distintas obras de conservación de suelo y agua en sus fincas, el tipo de obra está en dependencia de las condiciones agronómicas y topográficas de la

Estos agricultores no cumplen con los criterios de evaluación como para considerar que han adoptado la tecnología en sus fincas, esto no necesariamente significa que la adopción sea nula totalmente o que no estén en proceso de hacerlo, sino que ellos no cumplen con todos los criterios de evaluación dados por PASOLAC.

El $100 \%$ de los agricultores sujetos a estudio mantienen la práctica de las obras agronómicas y físicas de conservación de suelo, aumentando el área de cobertura de los SAF y realizando modificaciones en las tecnologías transferidas con el fin de adaptarlas a las condiciones que presta la finca.

La introducción de los SAF y el alto porcentaje de adopción en casi todos los municipios asegura una mejora en la producción de granos básicos, rompiendo con el sistema de agricultura tradicional y contribuyendo a la recuperación de los suelos en laderas secas de los municipios de León, Chinandega y Managua.

Obras agronómicas y físicas de conservación de suelos más utilizadas. La conservación de suelos representa junto con la mano de obra familiar, los recursos de producción más importantes con que cuenta el pequeño productor. El recurso

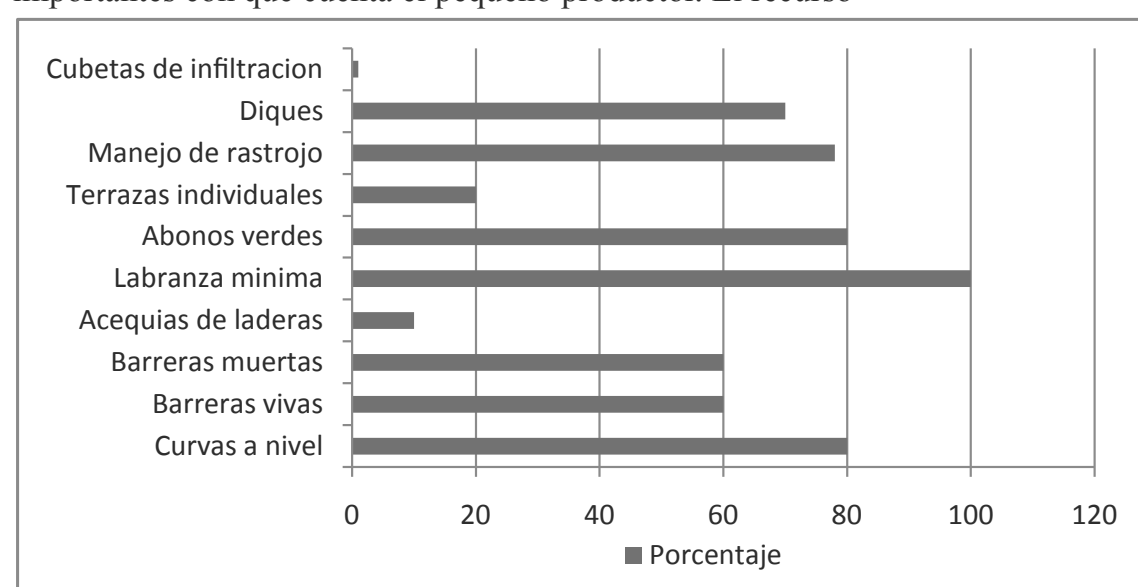

Figura 3. Obras agronómicas y físicas de conservación de suelos de mayor utilización. seguida por las curvas a nivel y la utilización de abonos verdes. La práctica de la NO QUEMA es muy utilizada entre los agricultores (80\%), siendo ésta una de las más importantes en la conservación de suelo evidenciándose que todos los agricultores que fueron sujetos de estudio tienen más de dos años de NO realizar la mala práctica de quema de potreros, luego le siguen las distintas obras de retención de agua y suelo, tales como diques, barreras vivas y muertas; variando éstas obras en función de la topografía del terreno y de las necesidades que tenga la parcela. Las obras menos utilizadas son: terrazas individuales y las acequias de laderas, sin embargo, la tecnología de cubas de infiltración no es utilizada por ninguno de los agricultores entrevistados, justificándose que por las condiciones de la finca, no es necesario este tipo de obra de conservación de suelo.

\section{CONCLUSIONES}

A tres años de iniciado el proceso de adopción y aceptación de los SAF se ha logrado que muchos de los agricultores de los municipios hayan adoptado y aceptado los SAF en sus fincas, a excepción de los municipios de San Francisco Libre donde aún hay mucho que trabajar para lograr el 100\% de adopción y aceptación así como en el municipio de El Jicaral que presenta un bajo índice de adopción.

Un alto porcentaje de los agricultores encuestados mantienen las tecnologías promovidas y estos, han expandido el sistema agroforestal promovido (GBAD y GBCM) en sus parcelas productivas, logrando así el manejo sostenible de la tierra.

El 73\% de la población encuestada ha logrado adoptar los sistemas productivos GBAD y GBCM, los agricultores se sienten satisfechos con los logros obtenidos y justifican la adopción porque finca, siendo la más utilizada la labranza mínima (100\%), 
dicen que han mejorado la producción al poseer suelos más fértiles y con mayor tiempo de humedad en el suelo.

Las obras agronómicas y físicas de conservación de suelo más utilizadas por los agricultores de todos los municipios son la labranza mínima, utilización de abonos verdes, las curvas a nivel y la no quema.

La inclusión de la mano de obra familiar es bastante alta, esto indica que el hombre o jefe de familia está consciente del apoyo que puede ofrecer la mujer y sus hijos, dejando atrás los prejuicios del machismo y a la vez minimiza el gasto por pago de mano de obra a un jornal ya que este indicador es la principal inversión que realizan los campesinos en su parcela.

\section{RECOMENDACIONES}

Es importante familiarizar a los agricultores con la necesidad de evaluar y monitorear el desarrollo de los sistemas agroforestales, con el fin de contar con información suficiente y necesaria en el momento de toma de decisiones, incorporación de cambios y adopción de nuevas tecnologías.

Institucionalizar los sistemas agroforestales como una alternativa válida, aunque no sea la única, para el manejo sostenible de terrenos agrícolas, áreas de protección, cuencas, zonas semi-áridas, etc. Es importante introducir y diseñar los sistemas conjuntamente con el productor y acompañarle de cerca en el manejo, introduciendo gradualmente nuevos elementos según su interés y capacidad.

El proyecto Manejo Sostenible de la Tierra debe monitorear cómo se realiza la distribución de recursos a los agricultores por parte de los organismos aliados, con la finalidad de asegurar que el recurso que le es entregado al productor, llegue de forma correcta y que su distribución sea uniforme al igual que la asistencia técnica.

Realizar un estudio de impacto o de sistematización al culminar el proyecto con la finalidad de profundizar en los logros obtenidos en los sistemas productivos de las familias campesinas con la introducción de estas tecnologías y obras de conservación de suelo.

Llevar un mejor control de la asistencia técnica dada a cada a uno de los agricultores para así, poder comparar la influencia del tiempo asignado a cada productor en la aceptación y adopción de una tecnología.

\section{REFERENCIAS BIBLIOGRAFÍCAS}

MARENA-PNUD (Ministerio de Ambiente y Recursos Naturales - Programa de Naciones Unidas para el Desarrollo). 2003. Programa de Acción Nacional de Lucha Contra la Desertificación y la Sequía. Managua, Nicaragua. 46 p.

Mora-Delgado, J; Díaz, M; Martínez, M; Holguin, VA. 2004. Un Método Rápido Para La Toma De Decisiones En Sistemas Agroforestales. (en línea). Consultado 01 de dic. de 2009. Disponible en http://www.fao.org/fileadmin/templates/lead/pdf/03_article04_es.pdf

PASOLAC; COSUDE (Programa para la Agricultura Sostenible en Laderas de Centro América; Agencia Suiza para el Desarrollo y la Cooperación). 2005. Guía técnica de conservación de suelo y agua. $1^{\text {a }}$ ed. San Salvador ES. 222 p.

Montagnini, F. 1992. Sistemas Agroforestales: Principios y Aplicaciones en los Trópicos. San José, Costa Rica. 622 p.

Ospina, A. 2007. Agroforestería; Aportes conceptuales, metodológicos y prácticos para el estudio de la Agroforestería. ACASOC. (en línea). Consultado 01 de ene de 2010. Disponible en http:/www.agroforesteriaecologica.com/index.php?section=5 